\title{
Relação entre cobertura pré-natal, características maternas, da gestação e APGAR dos recém nascidos vivos em Minas Gerais
}

\author{
Relationship between prenatal coverage, maternal, pregnancy and APGAR characteristics of live \\ newborn in Minas Gerais
}

Relación entre cobertura prenatal, características maternas, de embarazo y APGAR de recién nacidos vivos en Minas Gerais

\section{Resumo}

A saúde do recém-nascido sofre influência direta de múltiplos fatores maternos, como transformações físicas, emocionais, econômicas e sociais, idade materna, tipo de gestação, doenças prévias e número de consultas. Assim, a atenção pré-natal constitui-se de ações preventivas, diagnósticas, curativas e promotoras de saúde para a gestante e para o filho. O objetivo do artigo é analisar a influência dos fatores maternos, obstétricos e assistenciais nos valores encontrados na avaliação de APGAR dos recém-nascidos vivos de Minas Gerais. Este é um estudo epidemiológico transversal

${ }^{1}$ Discentes do Curso de Medicina do Centro Universitário de Patos de Minas- UNIPAM, MG-Brasil.

${ }^{2}$ Docentes do Curso de Medicina do Centro Universitário de Patos de Minas- UNIPAM, MG-Brasil. 
descritivo com dados obtidos por meio do DATASUS para obter informações a respeito dos nascidos vivos no estado de Minas Gerais entre 2015 e 2019. O estudo evidencia que a idade e a instrução materna possuem influência no número de consultas do pré-natal e o peso ao nascer e o APGAR estão relacionados com a efetividade da assistência obtida nas consultas. Conclui-se, portanto, que a efetividade do pré-natal é fundamental para prevenir e promover saúde para a mãe e para o bebê, devendo seguir as recomendações de frequência propostas pelo Ministério da Saúde para reduzir a morbimortalidade materna e infantil e melhorar a satisfação materna e os resultados perinatais, além de aumentar as chances do recém-nascido apresentar um APGAR adequado.

Palavras-chave: Pré-natal; Instrução materna; Idade materna; Peso ao nascer; APGAR.

\begin{abstract}
The newborn's health is directly influenced by multiple maternal factors, such as physical, emotional, economic and social changes, maternal age, type of pregnancy, previous illnesses and number of consultations. Thus, prenatal care consists of preventive, diagnostic, curative and health-promoting actions for the pregnant woman and for the child. The aim of the article is to show the relationship between prenatal coverage, maternal and pregnancy characteristics and the APGAR of live births. This is a descriptive cross-sectional epidemiological study with data obtained through DATASUS to obtain information about live births in the state of Minas Gerais. The study shows that age and maternal education influence the number of prenatal consultations, and birth weight and the APGAR are related to the effectiveness of the assistance obtained in the consultations. It is concluded, therefore, that the effectiveness of prenatal care is essential to prevent and promote health for the mother and baby, and should follow the frequency recommendations proposed by the Ministry of Health to reduce maternal and child morbidity and improve satisfaction maternal and perinatal outcomes, in addition to increasing the baby's chances of having an adequate APGAR.
\end{abstract}

Keywords: Prenatal care; Maternal instruction; Maternal age; Birth weight; APGAR.

\title{
Resumen
}

La salud del recién nacido está directamente influenciada por múltiples factores maternos, como cambios físicos, emocionales, económicos y sociales, edad materna, tipo de embarazo, enfermedades previas y número de consultas. Así, la atención prenatal consiste en acciones preventivas, diagnósticas, curativas y promotoras de la salud de la gestante y del niño. El objetivo del artículo es mostrar la relación entre la cobertura prenatal, las características maternas y del embarazo y la APGAR de los nacidos vivos. Se trata de un estudio epidemiológico descriptivo transversal con datos obtenidos a través de DATASUS para obtener información sobre nacidos vivos en el estado de Minas Gerais. El estudio muestra que la edad y la educación materna influyen en el número de consultas prenatales, y el peso al nacer y el APGAR se relacionan con la efectividad de la asistencia obtenida en las consultas. Se concluye, por tanto, que la efectividad de la atención prenatal es fundamental para prevenir y promover la salud de la madre y el bebé, debiendo seguir las recomendaciones de frecuencia propuestas por el Ministerio de Salud para reducir la morbilidad materna e infantil y mejorar la satisfacción de los resultados maternos y perinatales, además de aumentar las posibilidades del bebé de tener un APGAR adecuado.

Palabras clave: Prenatal; Instrucción materna; Edad materna; Peso de nacimiento; APGAR.

\section{Introdução}

O período gestacional vivenciado pela mulher traz consigo transformações físicas em seu corpo, bem como mudanças emocionais, econômicas e sociais (Oliveira, 2020). A saúde do recém-nascido sofre influência direta desses múltiplos fatores relacionados a esse período. Dentre os fatores estão os aspectos relacionados a características maternas, como a idade, o tipo de gestação e as doenças prévias apresentadas, assim como questões socioeconômicas e demográficas, além da adesão à assistência pré-natal (Pieroni, 2020).

Nesse âmbito, a atenção pré-natal constitui-se de um conjunto de ações que são, simultaneamente, preventivas, diagnósticas, curativas e promotoras de saúde, visando um bom desfecho da gestação para a mulher e o seu filho(Leal, Pereira, Viellas, Domingues \& Gama, 2020). Logo, uma melhoria na qualidade dessa assistência impacta diretamente na redução da morbimortalidade materna e infantil. Nesse contexto, é preconizado pelo programa Previne Brasil que as gestantes realizem, no mínimo, seis consultas de pré-natal, sendo a primeira delas efetuada até a $20^{\text {a }}$ semana gestacional. Dessa forma, a utilização de índices de adequação do pré-natal tem sido amplamente adotada para pesquisas em saúde pública, planejamento e alocação de recursos. Tal ação permitirá melhoria no acesso, na cobertura, na qualidade do acompanhamento à gestante, bem como na assistência ao parto, ao recém-nascido e à mulher no puerpério (Reis, Paiva, Ribeiro, Galvão \& Guedes, 2021).

Uma maneira eficaz de identificar a necessidade de implementação de novos programas de educação em saúde e de 
melhorias do cuidado perinatal, além de verificar o impacto nas intervenções de qualidade do serviço, é por meio do acompanhamento do índice de APGAR (Thomé et al., 2020).

Esse índice apresenta proporções úteis para a avaliação inicial do neonato, no primeiro, no quinto e, em determinadas condutas, no décimo minuto de vida. Esse escore avalia cinco sinais vitais, sendo eles o tônus muscular, a frequência cardíaca, a irritabilidade reflexa, a respiração e a cor da pele desse recém-nascido (T. B Silva et al., 2019).Cada um desses aspectos recebe notas individuais que, ao serem, somadas totalizam um valor que varia de zero a dez. Nesse viés, resultados maiores que sete são considerados adequados, enquanto valores inferiores configuram um sinal de alerta (L. S. R. Silva, Cavalcante, Carneiro, Oliveira, 2020).

Para tanto, se torna importante a realização e a interpretação dos valores do APGAR apresentado pelos recém-nascidos buscando relacioná-los com o número de consultas de pré-natal, bem como características maternas e gestacionais, pois servirão de subsídio para que sejam empreendidos esforços por parte dos serviços de saúde com a finalidade de contribuir para a redução da morbimortalidade materna e infantil. Portanto, o presente estudo objetiva analisar a influência desses fatores maternos, obstétricos e assistenciais nos valores encontrados na avaliação do índice de APGAR dos recém-nascidos vivos de Minas Gerais, entre 2015 e 2019.

\section{Metodologia}

Trata-se de um estudo epidemiológico transversal descritivo realizado no segundo semestre de 2021, com dados obtidos por meio do SINASC (Sistema de Informações sobre Nascidos Vivos) e pertencentes ao Departamento de Informática do Sistema Único de Saúde (DATASUS).

A coleta ocorreu no mês de outubro de 2021 e a partir dos dados foram organizadas tabelas para a realização da análise. A população do estudo incluiu todos os nascidos vivos filhos de mães residentes no estado de Minas Gerais no período entre2015 e 2019, últimos anos com dados disponíveis e confirmados.

Dentre os dados coletadas o número de consultas de pré-natal e a adequação de pré-natal, foram comparados com a idade da mãe, instrução da mãe, tipo de parto, peso ao nascer e APGAR de $1^{\circ}$ e $5^{\circ}$ minutos. Para o número de consultas de pré-natal foram consideradas as divisões: nenhuma, de 1 a 3 consultas, de 4 a 6 consultas, 7 ou mais consultas e as faixas correspondentes aos "ignorados" também foram utilizadas na análise. Para as demais variáveis, os itens "ignorados" também foram utilizados.

As variáveis foram analisadas por meio da criação de tabelas com as respectivas informações encontradas. Além disso, o estudo contou com o aporte de trabalhos acadêmicos entre os anos de 2015 e 2021. As principais bases de dados foram: National Library of Medicine (PubMed MEDLINE), Scientific Electronic Library Online (Scielo), Cochrane Database of Systematic Reviews (CDSR), Google Scholar, Biblioteca Virtual em Saúde (BVS) e EBSCO Information Services, no período compreendido entre agosto e setembro de 2021

Por se tratar da descrição e da análise apenas de informações de natureza secundária, não houve o contato com elementos referentes à identificação pessoal da população do estudo. Dessa maneira, não existiu a necessidade de aprovação pelo Comitê de Ética em Pesquisa (CEP).

\section{Resultados}

O Sistema de Informações sobre Nascidos Vivos (SINASC) registrou no estado de Minas Gerais (MG), entre 2015 e 2019, 1303316 nascidos vivos, dentre esses 13167 não tem registro de nenhuma consulta pré-natal, sendo que o restante foi dividido em categorias de 1 a 3 consultas, de 4 a 6 consultas, mais de 7 consultas e a parte que foi ignorada. 
Para análise das características sobre os nascidos vivos e o pré-natal em MG é necessário conhecer alguns dados, dentre eles os relacionados as gestantes, que possuem variáveis as quais podem elucidar um cenário sobre o desfecho do nascimento das crianças mineiras. Dessa forma, são apresentados os dados relacionados a idade materna (Tabela 1), a instrução materna (Tabela 2) e ao tipo de parto (Tabela 3), sendo que as duas primeiras variáveis estão correlacionadas com o número de consultas pré-natal realizadas, já a variável de tipo de parto é correlacionada com a adequação do pré-natal.

Os dados que expressam as maiores proporções em relação a idade materna encontram-se primeiramente na categoria de 1 a 3 consultas com a faixa etária de 20 a 24 anos, em contraste a isso a segunda proporção de maior destaque está situada na categoria de nenhuma consulta na mesma faixa etária da primeira proporção. Já as menores proporções em relação a idade materna estão localizadas nas categorias de 4 a 6 consultas e de 7 ou mais consultas, tais proporções são referentes a faixa etária de 60 a 64 anos.

Tabela 1: Relação entre a idade materna e o número de consultas de pré-natal dos nascidos vivos em Minas Gerais, entre 2015 e 2019.

\begin{tabular}{|c|c|c|c|c|c|c|c|c|c|c|c|c|}
\hline \multirow{2}{*}{$\begin{array}{c}\text { Idade da } \\
\text { mãe (anos) }\end{array}$} & \multicolumn{2}{|c|}{ Nenhuma } & \multicolumn{2}{|c|}{$\begin{array}{c}\text { De } 1 \text { a } 3 \\
\text { consultas }\end{array}$} & \multicolumn{2}{|c|}{$\begin{array}{c}\text { De } 4 \text { a } 6 \\
\text { consultas }\end{array}$} & \multicolumn{2}{|c|}{$\begin{array}{l}7 \text { ou mais } \\
\text { consultas }\end{array}$} & \multicolumn{2}{|c|}{ Ignorado } & \multicolumn{2}{|c|}{ Total } \\
\hline & $\mathrm{N}$ & $\%$ & $\mathrm{~N}$ & $\%$ & $\mathrm{~N}$ & $\%$ & $\mathrm{~N}$ & $\%$ & $\mathrm{~N}$ & $\%$ & $\mathrm{~N}$ & $\%$ \\
\hline 10 a 14 & 117 & 0,8 & 532 & 1,1 & 1954 & 0,8 & 4004 & 0,4 & 35 & 0,7 & 6642 & 0,5 \\
\hline 15 a 19 & 2106 & 15,9 & 9964 & 21,5 & 45215 & 18,7 & 115182 & 11,5 & 613 & 13,2 & 173080 & 13,2 \\
\hline 20 a 24 & 3409 & 25,8 & 13509 & 29,2 & 63541 & 26,3 & 219759 & 22,0 & 1110 & 24,2 & 301328 & 23,1 \\
\hline 25 a 29 & 3020 & 22,9 & 9614 & 20,8 & 54060 & 22,4 & 249635 & 25,0 & 1118 & 24,1 & 317447 & 24,3 \\
\hline 30 a 34 & 2497 & 18,9 & 6909 & 14,9 & 43193 & 17,9 & 236334 & 23,6 & 930 & 20,1 & 289863 & 22,2 \\
\hline 35 a 39 & 1525 & 11,5 & 4254 & 9,2 & 25882 & 10,7 & 140248 & 14,0 & 633 & 13,7 & 172542 & 13,2 \\
\hline 40 a 44 & 453 & 3,4 & 1303 & 2,8 & 6869 & 2,8 & 31233 & 3,1 & 168 & 3,6 & 40026 & 3,0 \\
\hline 45 a 49 & 33 & 0,2 & 98 & 0,2 & 408 & 0,1 & 1677 & 0,1 & 10 & 0,2 & 2226 & 0,1 \\
\hline 50 a 54 & 2 & 0,0 & 2 & 0,0 & 18 & 0,0 & 103 & 0,0 & 2 & 0,0 & 127 & 0,0 \\
\hline 55 a 59 & 2 & 0,0 & - & - & 2 & 0,0 & 18 & 0,0 & - & - & 22 & 0,0 \\
\hline 60 a 64 & - & - & 1 & 0,0 & 1 & 0,0 & 7 & 0,0 & - & - & 9 & 0,0 \\
\hline $\begin{array}{c}\text { Idade } \\
\text { ignorada }\end{array}$ & 3 & 0,0 & - & - & - & - & - & - & 1 & 0,0 & 4 & 0,0 \\
\hline Total & 13167 & 100 & 46186 & 100 & 241143 & 100 & 998200 & 100 & 4620 & 100 & 1303316 & 100 \\
\hline
\end{tabular}

Fonte: Ministério da Saúde/SVS - DASIS - Sistema de Informações sobre Nascidos Vivos (SINASC) (2021).

Além dessa variável, há a relação entre o nível de instrução da mãe com os números de consultas do pré-natal, sendo que as maiores proporções dessa correlação estão situadas em um único nível de instrução, de 8 a 11 anos, segue-se em ordem decrescente de porcentagem as categorias: de 4 a 6 consultas, de 1 a 3 consultas, de 7 ou mais consultas, nenhuma consulta, e a parcela ignorada. Em relação as menores proporções dessa variável, de forma decrescente, são apresentadas as categorias de 4 a 6 consultas e de 7 ou mais consultas, ambas proporções referentes a faixa de nível de nenhuma instrução.

Tabela 2: Relação entre a instrução materna e o número de consultas de pré-natal dos nascidos vivos em Minas Gerais, entre 2015 e 2019

\begin{tabular}{|c|c|c|c|c|c|c|c|c|c|c|c|c|}
\hline \multirow{2}{*}{$\begin{array}{c}\text { Instrução } \\
\text { da mãe } \\
\text { (anos) }\end{array}$} & \multicolumn{2}{|c|}{ Nenhuma } & \multicolumn{2}{|c|}{$\begin{array}{c}\text { De } 1 \text { a } 3 \\
\text { consultas }\end{array}$} & \multicolumn{2}{|c|}{$\begin{array}{c}\text { De } 4 \text { a } 6 \\
\text { consultas }\end{array}$} & \multicolumn{2}{|c|}{$\begin{array}{l}7 \text { ou mais } \\
\text { consultas }\end{array}$} & \multicolumn{2}{|c|}{ Ignorado } & \multicolumn{2}{|c|}{ Total } \\
\hline & $\mathrm{N}$ & $\%$ & $\mathrm{~N}$ & $\%$ & $\mathrm{~N}$ & $\%$ & $\mathrm{~N}$ & $\%$ & $\mathrm{~N}$ & $\%$ & $\mathrm{~N}$ & $\%$ \\
\hline Nenhuma & 141 & 1,0 & 303 & 0,6 & 911 & 0,3 & 1743 & 0,1 & 25 & 0,5 & 3123 & 0,2 \\
\hline 1 a 3 & 415 & 3,1 & 1303 & 2,8 & 4671 & 1,9 & 12990 & 1,3 & 118 & 2,5 & 19497 & 1,4 \\
\hline 4 a 7 & 2565 & 19,4 & 11106 & 24,0 & 43805 & 18,1 & 118205 & 11,8 & 843 & 18,2 & 176524 & 13,5 \\
\hline 8 a 11 & 7740 & 58,7 & 28987 & 62,7 & 155830 & 64,6 & 610290 & 61,1 & 2672 & 57,8 & 805519 & 61,8 \\
\hline 12 e mais & 1748 & 13,2 & 3751 & 8,1 & 32498 & 13,4 & 242958 & 24,3 & 834 & 18,0 & 281789 & 21,6 \\
\hline Ignorado & 558 & 4,2 & 736 & 1,5 & 3428 & 1,4 & 12014 & 1,2 & 128 & 2,7 & 16864 & 1,2 \\
\hline Total & 13167 & 100 & 46186 & 100 & 241143 & 100 & 998200 & 100 & 4620 & 100 & 1303316 & 100 \\
\hline
\end{tabular}

Fonte: Ministério da Saúde/SVS - DASIS - Sistema de Informações sobre Nascidos Vivos (SINASC) (2021). 
Ademais, foi necessário coletar dados em relação a adequação do pré-natal e o tipo de parto realizado, dessa forma as proporções de maior relevância foram encontradas na categoria de pré-natal mais do que adequado, sendo que o tipo de parto com maior proporção foi o Cesário seguido pelo Vaginal. Em relação as menores proporções estão situadas na categoria das gestantes que não fizeram pré-natal, sendo que o parto Cesário foi realizado em uma escala menor em relação a realização do parto Vaginal, além de constar que a categoria de parto ignorado situa-se em uma porcentagem intermediaria entre os dois tipos de partos já citados.

Tabela 3: Relação entre a adequação de pré-natal e o tipo de parto dos nascidos vivos em Minas Gerais, entre 2015 e 2019.

\begin{tabular}{ccccccccc}
\hline Adequação do & \multicolumn{2}{c}{ Vaginal } & \multicolumn{2}{c}{ Cesário } & \multicolumn{2}{c}{ Ignorado } & \multicolumn{2}{c}{ Total } \\
\cline { 2 - 9 } pré-natal & $\mathrm{N}$ & $\%$ & $\mathrm{~N}$ & $\%$ & $\mathrm{~N}$ & $\%$ & $\mathrm{~N}$ & $\%$ \\
\hline Não fez pré-natal & 2778 & 0,5 & 865 & 0,1 & 5 & 0,2 & 3648 & 0,2 \\
Inadequado & 100252 & 18,2 & 86671 & 11,5 & 246 & 13,3 & 187169 & 14,3 \\
Intermediário & 36989 & 6,6 & 33020 & 4,1 & 64 & 3,4 & 70073 & 5,3 \\
Adequado & 41308 & 7,4 & 46597 & 6,2 & 107 & 5,7 & 88012 & 6,7 \\
Mais que adequado & 338578 & 61,2 & 549030 & 73,3 & 1000 & 54,1 & 888608 & 68,1 \\
Não Classificados & 33207 & 6,0 & 32173 & 4,2 & 426 & 23,0 & 65806 & 5,0 \\
Total & 553112 & 100 & 748356 & 100 & 1848 & 100 & 1303316 & 100 \\
\hline
\end{tabular}

Fonte: Ministério da Saúde/SVS - DASIS - Sistema de Informações sobre Nascidos Vivos (SINASC) (2021).

Além disso, as condições do recém-nascido foram analisadas pelas variáveis peso ao nascer (tabela 4) e APGAR de $1^{\circ} \mathrm{e}$ $5^{\circ}$ minutos (tabela 5) de acordo com o número de consultas de pré-natal. Assim, as maiores proporções de baixo peso ao nascer, peso inferior a $2500 \mathrm{~g}$, situam-se nas categorias de nenhuma consulta de pré-natal e de 1 a 3 consultas, enquanto as menores proporções nas categorias de 4 a 6 consultas e de 7 ou mais consultas.

Tabela 4: Relação entre o peso ao nascer e o número de consultas de pré-natal dos nascidos vivos em Minas Gerais, entre 2015 e 2019.

\begin{tabular}{|c|c|c|c|c|c|c|c|c|c|c|c|c|}
\hline \multirow{2}{*}{$\begin{array}{c}\text { Peso ao } \\
\text { nascer (g) }\end{array}$} & \multicolumn{2}{|c|}{ Nenhuma } & \multicolumn{2}{|c|}{$\begin{array}{c}\text { De } 1 \text { a } 3 \\
\text { consultas }\end{array}$} & \multicolumn{2}{|c|}{$\begin{array}{c}\text { De } 4 \text { a } 6 \\
\text { consultas }\end{array}$} & \multicolumn{2}{|c|}{$\begin{array}{l}7 \text { ou mais } \\
\text { consultas }\end{array}$} & \multicolumn{2}{|c|}{ Ignorado } & \multicolumn{2}{|c|}{ Total } \\
\hline & $\mathrm{N}$ & $\%$ & $\mathrm{~N}$ & $\%$ & $\mathrm{~N}$ & $\%$ & $\mathrm{~N}$ & $\%$ & $\mathrm{~N}$ & $\%$ & $\mathrm{~N}$ & $\%$ \\
\hline 0 a 999 & 474 & 3,5 & 2107 & 4,6 & 4253 & 1,8 & 2043 & 0,2 & 153 & 3,3 & 9030 & 0,7 \\
\hline 1000 a 2499 & 2196 & 16,7 & 8149 & 17,7 & 33710 & 14,0 & 68335 & 6,9 & 820 & 17,8 & 113210 & 8,7 \\
\hline 2500 a 3999 & 10051 & 76,4 & 34700 & 75,1 & 196075 & 81,3 & 888640 & 89,0 & 4513 & 76,0 & 1132979 & 86,9 \\
\hline 4000 e mais & 417 & 3,2 & 1215 & 2,6 & 7087 & 2,9 & 39171 & 3,9 & 129 & 2,8 & 48019 & 3,7 \\
\hline Ignorado & 29 & 0,2 & 15 & 0,0 & 18 & 0,0 & 11 & 0,0 & 5 & 0,1 & 78 & 0,0 \\
\hline Total & 13167 & 100,0 & 46186 & 100,0 & 241143 & 100,0 & 998200 & 100,0 & 4620 & 100,0 & 1303316 & 100,0 \\
\hline
\end{tabular}

Fonte: Ministério da Saúde/SVS - DASIS - Sistema de Informações sobre Nascidos Vivos (SINASC) (2021).

Em relação aos valores de APGAR de $1^{\circ}$ minuto as maiores proporções da medida de 0 a 2 estão entre as categorias de nenhuma consulta de pré-natal e de 1 a 3 consultas e as menores estão nas categorias de 4 a 6 consultas e de 7 ou mais consultas. Enquanto as maiores proporções da medida de 8 a 10 estão representadas pelas categorias de 7 ou mais consultas e de 4 a 6 consultas e as menores proporções pelas categorias de nenhuma consulta e de 1 a 3 consultas. No que diz respeito aos valores de APGAR de $5^{\circ}$ minuto os padrões são semelhantes aos relatados no APGAR de $1^{\circ}$ minuto, uma vez que o maior percentual de medidas de 0 a 2 é representado pela categoria de 1 a 3 consultas e o menor pela categoria de 7 ou mais consultas, ao passo que o maior percentual de medidas de 8 a 10 é representado pela categoria de 7 ou mais consultas e o menor pela categoria de nenhuma consulta. 
Tabela 5: Relação entre o APGAR de $1^{\circ}$ e $5^{\circ}$ minutos e o número de consultas de pré-natal dos nascidos vivos em Minas Gerais, entre 2015 e 2019.

\begin{tabular}{|c|c|c|c|c|c|c|c|c|c|c|c|c|}
\hline PGAR & \multicolumn{2}{|c|}{ Nenhuma } & \multicolumn{2}{|c|}{ De 1 a 3 consultas } & \multicolumn{2}{|c|}{ De 4 a 6 consultas } & \multicolumn{2}{|c|}{7 ou mais consultas } & \multicolumn{2}{|c|}{ Ignorado } & \multicolumn{2}{|c|}{ Total } \\
\hline \multicolumn{13}{|c|}{ APGAR $1^{\circ}$ minuto } \\
\hline APGAR & $\mathrm{N}$ & $\%$ & $\mathrm{~N}$ & $\%$ & $\mathrm{~N}$ & $\%$ & $\mathrm{~N}$ & $\%$ & $\mathrm{~N}$ & $\%$ & $\mathrm{~N}$ & $\%$ \\
\hline 0 a 2 & 356 & 2,7 & 1278 & 2,8 & 3129 & 1,3 & 5884 & 0,6 & 115 & 2,5 & 10762 & 0,8 \\
\hline 3 a 5 & 609 & 4,6 & 2109 & 4,6 & 8278 & 3,5 & 23458 & 2,3 & 190 & 4,1 & 34644 & 2,7 \\
\hline 6 a 7 & 1223 & 9,3 & 4117 & 8,9 & 20563 & 8,5 & 71428 & 7,2 & 406 & 8,8 & 97737 & 7,5 \\
\hline 8 a 10 & 9911 & 75,3 & 36628 & 79,3 & 201187 & 83,4 & 870505 & 87,2 & 3642 & 78,8 & 1121873 & 86,1 \\
\hline Ignorado & 1068 & 8,1 & 2054 & 4,4 & 7986 & 3,3 & 26925 & 2,7 & 267 & 5,8 & 38300 & 2,9 \\
\hline Total & 13167 & 100,0 & 46186 & 100,0 & 241143 & 100,0 & 998200 & 100,0 & 4620 & 100,0 & 1303316 & 100,0 \\
\hline \multicolumn{13}{|c|}{ APGAR $5^{\circ}$ minuto } \\
\hline APGAR & $\mathrm{N}$ & $\%$ & $\mathrm{~N}$ & $\%$ & $\mathrm{~N}$ & $\%$ & $\mathrm{~N}$ & $\%$ & $\mathrm{~N}$ & $\%$ & $\mathrm{~N}$ & $\%$ \\
\hline 0 a 2 & 175 & 1,3 & 668 & 1,5 & 1160 & 0,5 & 1514 & 0,1 & 50 & 1,1 & 3567 & 0,3 \\
\hline 3 a 5 & 180 & 1,4 & 524 & 1,1 & 1417 & 0,6 & 2502 & 0,3 & 49 & 1,1 & 4672 & 0,4 \\
\hline 6 a 7 & 413 & 3,1 & 1352 & 2,9 & 4687 & 1,9 & 11073 & 1,1 & 121 & 2,6 & 17646 & 1,3 \\
\hline 8 a 10 & 11353 & 86,2 & 41641 & 90,2 & 226053 & 93,7 & 956497 & 95,8 & 4133 & 89,4 & 1239677 & 95,1 \\
\hline Ignorado & 1046 & 8,0 & 2001 & 4,3 & 7826 & 3,3 & 26614 & 2,7 & 267 & 5,8 & 37754 & 2,9 \\
\hline Total & 13167 & 100,0 & 46186 & 100,0 & 241143 & 100,0 & 998200 & 100,0 & 4620 & 100,0 & 1303316 & 100,0 \\
\hline
\end{tabular}

Fonte: Ministério da Saúde/SVS - DASIS - Sistema de Informações sobre Nascidos Vivos (SINASC) (2021).

\section{Discussão}

Conforme proposto pelo estudo, os dados dispostos na tabela denotam que o panorama epidemiológico dos recémnascidos e das gestantes e puérperas se mantém alinhados e diretamente proporcionais à escolaridade, à instrução e ao número de consultas de pré-natal. Assim sendo, de acordo com os resultados obtidos na Tabela 1, o número de nascidos vivos em Minas Gerais, entre 2015 e 2019, é maior na faixa etária materna de 25 a 29 anos, seguido pela faixa etária de 30 a 34 anos e 20 a 24 anos, posteriormente pela faixa etária de 35 a 39 anos e 15 a 19 anos, decrescendo a partir dos 40 anos de idade. Um estudo avaliou o perfil de nascimentos no estado de Minas Gerais no período de 2013 a 2017, constatando maior prevalência de nascidos vivos na faixa etária materna dos 20 aos 34 anos de idade (Alencar et al., 2020), o que vai ao encontro do que afirma Ferreira et al. (2018), dispondo de outro estudo realizado no município de Belo Horizonte que analisou, no período de 1994 a $2014,738.314$ nascimentos, sendo o maior número também na idade materna dos 20 aos 34 anos de idade. Em comparação com outro estado, uma pesquisa realizada no Piauí, de 2005 a 2014, converge com os resultados obtidos, demonstrando preponderância na mesma faixa etária (Santos et al., 2017). Entretanto, enquanto no presente estudo esse dado foi seguido pela faixa etária de 35 a 39 anos, a pesquisa realizada no Piauí registrou seguimento pela faixa etária menor que 20 anos.

Em contraponto, Sampaio, Rocha e Leal (2018), em seu estudo, pontuam que a média de idade das gestantes estudadas foi de 28 anos, e que, aproximadamente, 2,7\% das gestantes tinham idade maior ou igual a 35 anos. Nesse caso, é necessário estabelecer que a gravidez na adolescência e/ou após os 35 anos de idade tem sido relacionada ao baixo peso ao nascer, baixo índice de APGAR, prematuridade e maior ocorrência de partos cirúrgicos. Desse ponto de vista, é importante ressaltar que o desenvolvimento da gestação está diretamente relacionado com a idade, portanto, os riscos são maiores em idades mais precoces como também em idades mais tardias (Pereira \& Henriques, 2010). Considerando que atualmente a sociedade brasileira vive um período de "transição obstétrica", com maior envelhecimento da população obstétrica e menores taxas de fertilidade (Ferreira et al., 2018), decorrente da maior instrução escolar e inserção da mulher no mercado de trabalho, os números obtidos neste estudo refletem com maior veridicidade a realidade atual, uma vez que são dados do período de 2015 a 2019.

Ao analisar o número de consultas pré-natais segundo o grau de instrução materna (Tabela 2), nota-se maiores números correspondentes às mulheres que estudaram de 8 a 11 anos na coluna de 7 consultas ou mais. Em concomitância, uma pesquisa feita no município de Viçosa, de 2001 a 2007, constatou o mesmo resultado (Pereira \& Henriques, 2010). Além disso, o estudo 
realizado no estado de Minas Gerais do período de 2013 a 2017 também demonstrou maior prevalência em mulheres com instrução de 8 a 12 anos e de 7 ou mais consultas. Em concomitância, Santos, Abreu e Campos (2017), revelam dados de sua pesquisa nos quais mostram que a maioria das mulheres é alfabetizada, totalizando 75,1\% das participantes do estudo. Afirmam, nesse sentido, que a baixa escolaridade materna pode predispor a gestante a situações de risco, por estar associada ao baixo peso ao nascer, à prematuridade, mortalidade neonatal, mortalidade infantil, assim como ao aumento no número de gestações subsequentes, permeadas pelas mesmas condições.

Segundo a Organização Mundial de Saúde, quanto maior o número de contatos de assistência pré-natal, entre as gestantes e o prestador de atendimento de saúde, maior será a satisfação materna e melhores os resultados perinatais. Para isso, a OMS recomenda, no mínimo, oito contatos: um contato no primeiro trimestre, dois contatos no segundo trimestre e cinco contatos no terceiro trimestre, a depender do país. No Brasil, é consenso e recomendado pelo menos seis consultas de pré-natal OMS (2006).

Portanto, o número de mães com mais alto nível de escolaridade apresentou alta proporção de consultas de pré-natal e, consequentemente, melhores resultados na gestação, no parto e no puerpério (Pereira \& Henriques, 2010). Além disso, esses dados caracterizam a assistência ao pré-natal uma atividade acessível (Santos et al., 2017).

Nesse sentido, ao verificar a Tabela 3, a adequação do pré-natal de maior número foi a categoria do mais que adequado, e o tipo de parto mais realizado foi o cesáreo, seguido do parto vaginal. Resultado semelhante foi obtido pela pesquisa feita em Viçosa no ano de 2010, a qual constatou maior número de parto cesáreo e redução do parto vaginal (Alencar et al., 2020). Em contraste, o estudo realizado no município de Belo Horizonte apresentou predomínio do parto vaginal, mas com aumento progressivo do índice de cesáreas (Ferreira et al., 2018). Também, o estudo realizado no Piauí em 2017 demonstrou uma taxa maior de partos por via vaginal, seguido dos partos por via cesárea (Santos et al., 2017). Entretanto, as taxas de cesáreas encontradas em todos os estudos citados e neste presente estudo estão acima do 10\% recomendado pela OMS.

Ao avaliar o peso ao nascer (Tabela 4), constatou-se maior prevalência de recém-nascidos vivos com peso adequado, de 3000 a 3999g. O peso ao nascer é uma medida de crescimento intrauterino que influencia a sobrevida da criança, um importante indicador geral do nível de saúde da população estudada, pois reflete condições socioeconômicas (Pereira \& Henriques, 2010). Além disso, o peso ao nascer está relacionado com a efetividade da assistência pré-natal, uma vez que números maiores de nascidos com peso adequado estão encontrados na coluna de 7 ou mais consultas. Um estudo semelhante, realizado em Minas Gerais no período de 2013 a 2017, obteve resultados parecidos, com maior ocorrência de nascidos com peso adequado (Alencar et al., 2020).

Ao analisar o índice de APGAR, avaliado no $1^{\circ}$ e $5^{\circ}$ minuto (Tabela 5), este estudo demonstrou que em ambos a maior prevalência foi vista no índice de 8 a 10. Além disso, é possível perceber que os valores do índice de APGAR crescem à medida em que se aumenta o número de consultas de pré-natal, sendo os maiores valores obtidos nas colunas de 7 consultas ou mais. Resultado semelhante foi obtido em um estudo que analisou o pré-natal e APGAR no $1^{\circ}$ minuto de nascidos vivos em 2018, chegando à conclusão de que um pré-natal acessível e adequado determina um bom prognóstico para o neonato e também contribui para a diminuição da taxa de mortalidade perinatal (Thomé et al., 2020). Além desse, a pesquisa realizada no estado de Minas Gerais no período de 2013 a 2017 constatou o mesmo resultado, de maior prevalência no índice de 8 a 10 minutos em ambos os índices (Alencar et al., 2020).

\section{Conclusão}

Na tentativa de analisar a influência dos fatores maternos, obstétricos e assistenciais nos valores encontrados na avaliação do índice de APGAR dos recém-nascidos vivos de Minas Gerais, entre 2015 e 2019, foi possível concluir que: 25 a 29 anos foi a maior faixa etária materna encontrada; traçou-se uma relação do nível mais alto de escolaridade materna com a maior proporção de consultas de pré-natal, gerando melhores resultados na gestação, no parto e no puerpério; além disso, o tipo de parto mais 
realizado foi o cesáreo e, por fim, ao comparar o peso ao nascer e os índices de APGAR, constatou-se maior prevalência de recém-nascidos vivos com peso adequado e com o APGAR de 8 a 10. Todas as variáveis estão relacionadas com a efetividade da assistência pré-natal, vez que os índices são diretamente proporcionais ao número de consultas da mãe.

Em função da relevância da temática em questão, faz-se necessária a constante reavaliação dos dados em questão para uma análise mais aprofundada e atualizada dos parâmetros discutidos. Dessa forma, tal fato configura-se como uma medida capaz de promover condutas e ações que possibilitem a redução dos índices de morbimortalidade do binômio materno-fetal.

Conclui-se, portanto, que as características maternas, bem como a adesão à assistência pré-natal estão diretamente relacionados com a saúde do recém-nascido e com as suas condições imediatas ao nascimento, registradas pelo APGAR. Logo, o pré-natal é um instrumento de fundamental importância para reduzir a morbimortalidade materna e infantil e melhorar a satisfação materna e os resultados perinatais, devendo seguir as recomendações de quantidade de consultas e frequência propostas pelo Ministério da Saúde.

\section{Referências}

Silva, T. B., Dantas, A. J. M., No 3º, Moreira, A. C., Santos, V. M. P. R., Sousa, J. O., Frota, M. C. Q. A., Carneiro, J. K. R., Oliveira, M. A. S. (2019). Índice de Apgar relacionado a Fatores Maternos/Obstétricos e Neonatais. Revista Prevenção de Infecção em Saúde, 5:8389.

Leal, M. C., Pereira, A. P. E., Viellas, E. F., Domingues, R. M. S. M., Gama, S. G. N. (2020). Prenatal care in the Brazilian public health services. Revista de saude publica, 54, 08 .

Oliveira, D. N. (2020). Desfechos obstétricos em gestações tardias no estado de Minas Gerais. Revista Eletrônica Acervo Enfermagem, 3, 2555. Reduperado de https://acervomais.com.br/index.php/enfermagem/article/view/2555/1502

Pieroni, L. C. (2020). O impacto da realização do pré-natal nas condições clínicas de nascimento dos recém-nascidos atendidos no hospital de são luiz em cáceres - MT( Dissertação de Mestrado).Universidade Brasil, São Paulo, SP.

Reis, S. N., Paiva, I. G., Ribeiro, L. C. C., Galvao, E. L., Guedes, H. M. (2021). Adequação da assistência ao pré-natal para mulheres do Vale do Jequitinhonha, Minas Gerais - Brasil. Mundo da Saude, 1(45), 130-139.

Silva, L. S. R., Cavalcante, A. N., Carneiro, J. K. R., Oliveira, M. A. S. (2020). Índice de Apgar correlacionado a fatores maternos, obstétricos e neonatais a partir de dados coletados no Centro de Saúde da Família do bairro Dom Expedito Lopes situado no município de Sobral/CE. Revista Científica da Faculdade de Medicina de Campos, 15 (1), 25-30.

Thome, M. T., Amaral, G. R., Miranda, C. C., Amaral, L. M., Miranda, S. S., Ramos, R. S., R, B. C. E., Campelo, G. Q. (2020). Análise Do Pré-Natal E Do Apgar No $1^{\circ}$ Minuto De Nascidos Vivos Em 2018. Brazilian Journal of Development, 6(8), 54384-54392.

Pereira. A. C., Henriques, B. D. (2010, 05 de Maio). Perfil dos nascidos vivos em Viçosa, Minas Gerais, no período de 2001 a 2007. Ver Med Minas Gerais, 20(4), p.508-513.

Alencar, N. P. F. C., Castro, J. M., Martins, R. E. C., Azevedo, M. A., Oliveira, T. V. C., Proti, E. S., Araujo, D. A., Arruda, J. S. D., Guerra, C. H. W., Costa, W. J. T. (2020, Fevereiro). Perfil de nascimentos no estado de Minas Gerais no período de 2013 a 2017. Revista Eletrônica Acervo Enfermagem, 4.

Santos, G. M., Silva, A. J. A., Costa, R. F., Silva, L. A., Sousa, C. R. O., Barreto, M. T. S. (2017, 18 de Setembro). Análise do perfil das puérperas e dos nascidos vivos em um estado do nordeste brasileiro. Revista UNINGÁ Review, 31(1), p.12-18.

Thomé, M. T., Amaral, G. R., Miranda, C. C., Amaral, L. M., Miranda, S. S., Ramos, R. S., Rezende, B. C. E., Campelo, G. Q. (2020, 05 de Agosto). Análise do pré-natal e do Apgar no $1^{\circ}$ minuto de nascidos vivos em 2018. Brasilian Journal of Development, 6(8), p. 54384-54392.

Ferreira, V. A., Gomes, N. S., Pissetti, C. W., Silva, S. R., Ruiz, M. T. (2018). Perfil epidemiológico dos nascidos vivos em Belo Horizonte, MG, Brasil. Revista de Enfermagem do Centro-Oeste Mineiro, (8), p.2550.

Organização Mundial da Saúde. (2006). Recomendações da OMS sobre atendimento pré-natal para uma experiência gestacional positiva: Resumo.

Sampaio, A. F. S., Rocha, M. J. F. da, Leal, E. A. S. (2018). High-risk pregnancy: clinical-epidemiological profile of pregnant women attended at the prenatal service of the Public Maternity Hospital of Rio Branco, Acre. Revista Brasileira de Saúde Materno Infantil, 18(3), 559-566.

Santos, T. M. M. G., Abreu, A. P. S. B., Campos, T. G. Avaliação dos registros no cartão de pré-natal da gestante. Revista de Enfermagem UFPE on line, 11(7), 2939-2945. doi:https://doi.org/10.5205/1981-8963-v11i7a23474p2939-2945-2017. 